\title{
拔气管导管后生理盐水润喉对于缓解患者术后 咽痛的效果分析
}

\section{Analysis of the effect of normal saline throat moistening after tracheal catheter extraction on relieving patients with postoperative pharynx pain}

\author{
杜万秋 刘丹丹 邱晓东 \\ Wanqiu Du Dandan Liu Xiaodong Qiu \\ 东南大学附属中大医院麻醉科 中国 ・江苏 南京 210009 \\ Department of Anesthesiology, Zhongda Hospital, Southeast University, Nanjing, Jiangsu, 210009, China
}

摘 要: 目的: 观察拔除气管导管后生理盐水润喉对于全麻患者术后咽痛的临床效果。方法: 254 例全身麻醉气管插管后进 入麻醉恢复室的患者,记录气管插管后咽痛发生率。对咽痛患者随机予以生理盐水 $10 \mathrm{ml}$ 润喉或不做处理, 记录 $10 \mathrm{~min}$ 之后患 者咽痛情况并于术后第二日行麻醉满意度调查。结果: 术后咽痛发生率 $29 \%$,较对照组相比,生理盐水组患者咽痛明显改善 $(\mathrm{P}<0.05)$ 。结论: 全麻拔气管导管后予以生理盐水 $10 \mathrm{ml}$ 润喉, 能有效缓解术后咽痛, 操作简单易行无并发症。

\begin{abstract}
Objective: To observe the clinical effect of normal saline laryngopharynx after tracheal catheter removal on postoperative pharyngopharyngia in patients under general anesthesia. Methods: The incidence of pharynx pain after tracheal intubation was recorded in 254 patients who entered the anesthesia recovery room after general anesthesia. Patients with pharyngeal pain were randomly given $10 \mathrm{ml}$ of normal saline or no treatment. The pharyngeal pain of the patients was recorded 10min later, and anesthesia satisfaction survey was conducted on the second day after surgery. Results: The incidence of postoperative pharynx pain was $29 \%$, compared with the control group, the pharynx pain in the normal saline group was significantly improved $(\mathrm{P}<0.05)$. Conclusion: Using normal saline $10 \mathrm{ml}$ to moisten the throat after tracheal catheter withdrawal under general anesthesia can effectively relieve postoperative pharynx pain, and the operation is simple and easy without complications.
\end{abstract}

关键词: 生理盐水; 全身麻醉;拔气管导管后咽痛

Keywords : normal saline general anesthesia pharyngias after tracheal tube withdrawal

DOI : $10.36012 /$ pmr. v2i6. 2965

全身麻醉时行气管插管是一项侵袭性操作, 插气管导 管、拔气管导管及口腔内吸引操作等容易引起术后咽痛。国 外研究报道术后咽痛发生率约为 30\% 70\% ${ }^{[1]}$, 而国内研究 表明术后咽痛发生率在麻醉恢复室 (PACU) 可高达 50\%, 甚 至在术后 $24 \sim 30 \mathrm{~h}$ 内仍可观察到较明显的咽痛 ${ }^{[2]}$ 。普遍观 念认为气管插管引起咽痛无法避免, 且程度多不剧烈, 也并 不影响患者手术预后, 因此该并发症多为麻醉医生及外科医 生所忽视。然而我们在术后随访中发现全麻后咽痛是病人
除切口疼痛外非常多见的一项主诉, 影响患者术后早期饮 食, 影响患者术后满意度及舒适度。因此, 为了改善术后咽 痛这一全麻并发症, 我科在 PACU 拔气管导管后即刻, 给予 患者生理盐水润喉治疗术后咽痛, 取得了较满意的效果, 现 报告如下。

\section{1 资料与方法}

\section{1 一般资料}

【作者简介】杜万秋, 女, 汉, 江苏邳州人, 护师, 学士, 主要从事麻醉护理方向。 
我科是一所综合性三级甲等医院重点科室, 共开放手术 间 25 间,年手术量约 5 万台,复苏床位数 14 张, 年复苏量约 3 万台, 现麻醉护士 17 名 (主管护师 3 名、护师 12 名、护士 2 名), 主要工作岗位有: 麻醉后复苏、胃镜室麻醉配合和复苏、 术后疼痛管理、药品耗材管理。记录我院 2019 年 12 月 1 日 至 12 月 31 日经气管插管行全身麻醉并在术后进入 $\mathrm{PACU}$ 的患者 254 例。ASA 分级 I II 级; 年龄 18 65 岁; 患者均无 精神疾病; 无沟通障碍; 所有患者意识清醒; 术前均严格执行 禁食禁水;所有气管插管操作均一次完成; 所有患者术后无 严重并发症。患者一般情况无统计学差异。本研究经本院 伦理委员会批准,所有患者均知情同意并自愿参与。

\section{2 研究方案}

所有患者在 PACU 拔气管导管后, 由一随访专项人员进 行咽痛评估并记录。所有的患者在拔气管导管后评估 steward 评分达到 6 分, 由 PACU 医生随机予以患者生理盐水 $10 \mathrm{ml}$ 润喉或者不做处理。10min 后, 由上述同一随访专项人 员在不知道患者接受何种处理的情况下, 再次行咽痛评估并 记录,并于术后第二日随访记录患者麻醉满意度。

\section{3 统计学研究}

本研究所获取的数据均接受 SPSS22.0 软件进行统计学 分析, 分类资料采用卡方检验, 当 $\mathrm{P}<0.05$ 时认为有统计学 意义。

\section{2 结果}

研究共纳入患者 254 人,拔气管导管后咽痛 74 人, 无咽 痛 180 人。我科 PACU 咽痛发生率 $29 \%$ 。患者一般情况如 表 1 。

\section{表 1 患者一般资料}

\begin{tabular}{c|c|c|c}
\hline 组别 & 例数 $(\%)$ & 性别 (男/女) & 年龄 $(\mathrm{x} \pm \mathrm{s})$ \\
\hline 咽痛患者 & $74(29 \%)$ & $36 / 38$ & $41.6 \pm 13.3$ \\
\hline 无咽痛患者 & $180(71 \%)$ & $92 / 88$ & $47.5 \pm 13.4$ \\
\hline
\end{tabular}

咽痛组患者随机予以生理盐水干预观察, 结果见表 2

\section{表 2 患者生理盐水干预观察}

\begin{tabular}{c|c|c|c}
\hline 组别 & 无效 & 有效 & 合计 \\
\hline 观察组 & 10 & 29 & 39 \\
\hline 对照组 & 19 & 16 & 35 \\
\hline$\chi^{2}$ & & & 6.35 \\
\hline$P$ & & & $<0.05$ \\
\hline
\end{tabular}

患者麻醉满意度见表 3

\section{表 3 患者满意度调查}

\begin{tabular}{c|c|c|c}
\hline 组别 & 满意 & 不满意 & 合计 \\
\hline 观察组 & 36 & 3 & 39 \\
\hline 对照组 & 20 & 15 & 35 \\
\hline$\chi^{2}$ & & & 8.15 \\
\hline$P$ & & & $<0.05$ \\
\hline
\end{tabular}

\section{3 讨论}

全麻气管插管后近 $20 \%$ 的患者出现了咽痛,程度虽不剧 烈,但让患者产生了不愉快的体验并影响了麻醉满意度。国 外研究显示, 气管插管操作本身、女性、年轻、术前患有肺部 疾病、长时间气管插管状态、特殊体位均是术后咽痛的高危 因素 ${ }^{[3]}$ 。目前国内外对于术后咽痛的治疗仍未达成共识, 较 多的预防方法为导管涂抹利多卡因及口咽部表麻,该方法有 一定效果, 缺陷在于作用时间不够长及局麻药表麻时口感苦 澀患者接受度不高。因此,探寻一种有效且合理易行的防治 办法是临床治疗及护理关注的焦点。本研究证实了生理盐 水干预组患者咽痛明显改善 $(\mathrm{P}<0.05)$, 表明该方法行之有 效,并且未见任何明显不良反应。本研究的优点: (1) 操作简 单易行, 低成本成效大。(2) 患者接受度高, 易理解, 易配合, 无创伤。(3)生理盐水清洁口腔, 预防感染, 助于术后康复。 (4) 提高患者舒适度和麻醉满意度。

综上所述,拔气管导管后患者清醒且配合, 评估 steward 评分达到 6 分,给予生理盐水 $10 \mathrm{ml}$ 润喉可有效改善患者咽 痛,提高全麻满意度。

\section{参考文献}

[1] Tanaka Y, Nakayama T, Nishimori M, et al. Lidocaine for preventing postoperative score throat. Cocharane Database Syst Rev, 2015,14(7): CD004081.

[2] 张点, 刘俊, 吴静. 201 例气管内插管术后咽痛、声嘶发生率调 查. 宁夏医学杂志, 2010,10(32):932-933.

[3 ] El - Boghdadly K, Bailey CR, Wiles MD. Postoperative sore throat: a systematic review. Anesthesia, 2016,71:706-717. 